\title{
VII. Normen und Praxis ärztlichen Handelns
}

Die Beziehungen zwischen Ärzten und ihren Patienten sind für die Beschaffenheit einer medikalen Kultur ähnlich bedeutsam wie die Menge der verfügbaren medizinischen Ressourcen und der Modus ihrer Verteilung. Die Frage nach Kontinuität und Wandel in diesen Beziehungen lenkt den Blick auf das Feld des medizinischen Alltagshandelns, desjenigen Bereichs ärztlicher Tätigkeit, der als „Normalmedizin" von weiten Teilen der Bevölkerung erfahren werden konnte. Auch wenn man einwenden kann, daß immer dann, wenn im „Dritten Reich“ von „Normalmedizin“ die Rede ist, auch diejenigen Kranken mitzudenken sind, für die es keine reguläre medizinische Behandlung mehr gab - Juden, als „erbkrank“ verfemte, Zwangsarbeiter und in den letzten Kriegsjahren zunehmend auch alte und chronisch kranke Patienten ${ }^{1}$-, ist der Blick auf das ärztliche Alltagshandeln unerläßlich, denn Medizin war nicht nur als Element des nationalsozialistischen Terrorregimes in den Herrschaftsalltag des "Dritten Reiches“ eingebunden, sondern auch als Bestandteil einer Normalität, die das Funktionieren und Weiterbestehen dieser Herrschaft erst ermöglichte. Hier trafen nationalsozialistischer Umgestaltungswille und ein festgefügtes ärztliches Rollenverständnis aufeinander. Die Frage nach Kontinuität und Wandel in den Arzt-Patienten-Beziehungen ist daher auch eine Frage nach der Eindringtiefe des Nationalsozialismus in die deutsche Gesellschaft.

$\mathrm{Daß}$ man bislang nur wenig über die Arzt-Patienten-Beziehungen während der NS-Herrschaft weiß, resultiert zum Teil daraus, daß sich dieser intime Bereich dem Historiker ungleich schwerer erschließt als die politisch gesetzten Standards der medizinischen Versorgung. Zwar fehlt es nicht an Belegen für den Willen des Regimes, gerade diesem Bereich seinen Stempel aufzuprägen, ${ }^{2}$ doch sind Quellen, die einen direkten Einblick in die Praxis ärztlichen Handelns erlauben, ausgesprochen selten. ${ }^{3}$ Einige Hinweise, wie Patienten in der NS-Zeit ärztliches Handeln wahrnahmen, geben die Berichte des Sicherheitsdienstes der SS, der ein wachsames Auge darauf hatte, wie die Deutschen über ihr Gesundheitswesen dachten. Die meisten ärztlichen Selbstzeugnisse aus der ersten Hälfte des 20. Jahrhunderts blenden diesen Aspekt medizinischer Berufsausübung dagegen aus. Dort, wo die Arzt-Patienten-Beziehung zum Gegenstand von Verwaltungshandeln wurde und sich in Behördenakten niederschlug, hat man es zumeist mit Extremfällen zu tun, etwa dann, wenn sich ein Patient beschwerdeführend an die Medizinalverwaltung wandte, einen Arzt, was sehr selten vorkam, juristisch belangte, oder wenn ärztliches Handeln aus der Sicht des Regimes tadelnswürdig war.

1 Vgl. Jäckle, Pflicht, S. 59f.; für die jüdischen Patienten Kater, Medicine, S. 409 f.; für die Zwangsarbeiter Heusler, Ausländereinsatz, S. 344-386.

2 So etwa das als Programmschrift angelegte Buch des Schulungsreferenten im Hauptamt für Volksgesundheit, Rudolf Ramm, Rechts- und Standeskunde, das "dem Studierenden der Medizin und dem Jungarzt Weggenosse und Wegweiser" (S. VI) sein wollte.

3 Kudlien, Fürsorge, S. 99 f. 
Ein Versuch, ärztliches Handeln in den Jahren der nationalsozialistischen Herrschaft zu erfassen, sollte drei Problemebenen unterscheiden: die intentionale Ebene medizinethischer und professioneller Normen, die die Zielrichtung der vom NS-Regime angestrebten Veränderungen markierten, die Ebene tatsächlich realisierter Veränderungen in der medizinischen Praxis und die Ebene der Wahrnehmung und Verarbeitung ärztlichen Handelns durch die Patienten.

Die angestrebten Veränderungen auf der intentionalen Ebene medizinethischer und professioneller Normen sind leicht zu greifen. „Der deutsche Arzt soll Volksführer sein! “4 Dieses Postulat des Reichsärzteführers Wagner konnten sich viele der in der Zwischenkriegszeit arg gebeutelten Mediziner leicht zu eigen machen. Das nationalsozialistische Konzept der Gesundheitsführung rückte den Arzt als erbbiologischen Experten eng in die Nähe der Staatsmacht, unterstrich seine bevorzugte Stellung gegenüber anderen Heilberufen und gab seiner ins Wanken geratenen Autorität gegenüber dem Patienten neuen Halt. ${ }^{5}$ Der neue deutsche Arzt sollte, so der Reichsärzteführer Wagner auf dem Parteitag 1937, „den deutschen Menschen nicht nur körperlich betreuen, sondern auch seelisch [...] führen nach den Glaubenssätzen unserer nationalsozialistischen Weltanschauung ". ${ }^{6}$ Der entscheidende Punkt liegt weniger im Entwurf einer hierarchischen Arzt-PatientenBeziehung, die in den Reden Wagners zum Ausdruck kommt. Diese Vorstellung war durchaus zeitgenössisch. Vielmehr markiert die Unterordnung der Arzt-Patienten-Beziehung unter die Glaubenssätze der nationalsozialistischen Weltanschauung den Kern der angestrebten Veränderung eines Verhältnisses, das Ärzte bislang gerade durch seinen apolitischen Charakter definiert hatten. ${ }^{7}$ Gegen den "Wahn von der Gleichheit" 8 und die am medizinischen Bedarf orientierte Gesundheitsversorgung setzten die Nationalsozialisten ein Konzept medizinischer Versorgung, das auf der "naturgegebenen Ungleichheit der Menschen“ basierte und Gesundheitsressourcen nach rassistischen Wertigkeitskriterien und gesellschaftlichen Nützlichkeitskalkülen zuteilte. Nicht mehr der einzelne Mensch und sein kranker Körper standen im Mittelpunkt dieser Medizin, sondern „das Recht und die Notwendigkeiten des gesamten Volkes". ${ }^{9}$ Die Heiler-Identität des Arztes wurde auf diese Weise durch neue, machtvolle Attribute erweitert und teilweise beiseite geschoben. Ärztliches Handeln sollte nicht mehr von medizinischen Rationalitätskriterien geleitet werden, sondern in erster Linie von den Vorgaben des nationalsozialistischen Staates. Der "Gesundheitsoffizier", der "Priesterarzt" der Neuen Deutschen Heilkunde, der „Erbheger" im Gesundheitsamt und der

4 G. Wagner, Die Stellung des Arztes im Neuen Deutschland. Vortrag auf dem 3. Internationalen Kongress für das ärztliche Fortbildungswesen in Berlin, in: Ziel und Weg 7 (1937), S. 394-397, 396.

5 Zur sogenannten „Krise der Medizin“ vgl. Wolf, Interessen, S. 124-130.

6 G. Wagner, Ziele der nationalsozialistischen Gesundheitspolitik. Rede Wagners auf dem Reichsparteitag der NSDAP 1937, in: Ziel und Weg 7 (1937), S. 493.

7 Thomsen, Ärzte, S. 63.

8 Unser Reichsärzteführer spricht. Rede Gerhard Wagners auf dem Reichsparteitag 1935, in: Ziel und Weg 5 (1935), S. 431-438, 431.

9 Unser Reichsärzteführer spricht. Rede Gerhard Wagners auf dem Reichsparteitag 1936, in: Ziel und Weg 6 (1936), S. 509-515, 512. 
„Volksarzt“, der an die Stelle des überholten Individualarztes treten sollte ${ }^{10}$ - alle diese Begriffe sind Chiffren für ein gesundheitspolitisches Konzept, das den Arzt über den Patienten stellte und ihn gleichzeitig zum Werkzeug eines Gesundheitssystems machte, das das Wohl des einzelnen Kranken den Interessen der nationalsozialistischen "Volksgemeinschaft" unterordnete. Von diesem Standpunkt aus konnte auch ärztliches Handeln gerechtfertigt sein, das nicht mehr der Hilfe für den einzelnen Kranken diente, sondern den übergeordneten Zwecken des „Volkskörpers" nützlich war, so daß der Arzt bei einer Kollision beider Zielsetzungen leicht zum Täter an seinem Patienten werden konnte. ${ }^{11}$ Bei Bedarf, so ein Vertreter der Rüstungswirtschaft, hätte der Arzt „den Arbeiter auch einmal gegen sein medizinisches Gewissen davon zu überzeugen, daß er stark genug für die Arbeit sei“.12 Und in letzter Konsequenz konnte eine am "Volkskörper" orientierte medizinische Standesethik während des Krieges unter Ärzten für die Krankenmorde werben, indem sie die wirtschaftliche Belastung der Allgemeinheit durch Geisteskranke herausstellte. ${ }^{13}$

Die hier skizzierten Veränderungen auf der Ebene der standespolitischen und medizinethischen Diskussion lassen freilich nur sehr bedingt Schlüsse darüber zu, wieweit solche Konzepte in die tägliche Praxis ärztlichen Handelns vordrangen. Gerade die Behandlung der Fälle, in denen Mediziner erkennbar gegen tradierte ärztliche Standespflichten verstießen, zeigt, daß sich das Arzt-Patienten-Verhältnis nicht ohne weiteres unter das Diktat des Politischen stellen ließ. Wenige Monate vor dem Beginn des Zweiten Weltkrieges verurteilte der Reichsärztegerichtshof einen Arzt, der einen schwer mißhandelten Arbeitslagerinsassen nur oberflächlich untersucht hatte, zu einer empfindlichen Geldstrafe. Der Urteilstenor rügte den „erschreckenden Mangel an Pflichtgefühl und Verantwortungsbewußtsein“ des verurteilten Arztes. Dieser sei „wie nur irgendeines geeignet [...] das Ansehen des ärztlichen Standes und das Vertrauen zur Ärzteschaft zu untergraben“. 14

Seit September 1939 prägten kriegsbedingte Veränderungen die Arzt-Patienten-Beziehung. Zuvorderst wurde ärztlicher Rat knapper und damit wertvoller. Anfang 1944 standen der Zivilbevölkerung im Durchschnitt etwa halb so viele Ärzte zur Verfügung wie vor Kriegsbeginn, wobei der Ärztemangel in den ländlichen Gebieten teilweise noch weitaus gravierendere Formen annahm als in dichter

10 Ramm, Rechts- und Standeskunde, S. 80.

11 Bei den hier zugespitzt markierten Veränderungslinien darf freilich nicht außer acht gelassen werden, daß zentrale Bereiche des ärztlichen Verhaltenskodex weiterhin Bestand hatten. Auch nationalsozialistische Programmschriften betonten die konstitutive Bedeutung der Vertrauensbasis für die Arzt-Patienten-Beziehung, seine Sorgfaltspflichten gegenüber dem Patienten und die Bedeutung der ärztlichen Schweigepflicht; Ramm, Rechts- und Standeskunde, S. 96, 100, 112 f.

12 Aktenvermerk vom 13. 4. 1937, BA-MA, Wi I F 5/1223-1, Bl. 2., zit. nach Wuttke, Ideologien, S. 170.

13 Ramm, Rechts- und Standeskunde, S. 103 f.; dagegen allerdings entschieden der aus einer Einführungsvorlesung entstandene Abriß des Göttinger Klinikers Gruber, Ethik, S. 2729; für diese Position vgl. auch Kersting, Anstaltsärzte, S. $322 \mathrm{f}$.

14 Urteil des Ärztegerichtshofes gegen Hans F., 13. 1. 1939, AKBV/135. 
besiedelten Regionen. ${ }^{15}$ Vor allem jedoch gewann das ärztliche Urteil Einfluß auf immer größere Lebensbereiche, da Wirtschaftsämter und Händler die Zuteilung knapper Güter in zunehmendem Maße von einem medizinisch begründeten Bedarf abhängig machten und ,jede kriegsnotwendige Einschränkung auf irgendeinem Gebiet sofort dazu führ[t]e, daß die Bevölkerung “16 sich „zur Umgehung der Einschränkung [...] zwecks Ausstellung von Bescheinigungen an die Ärzte“ wandte. Anfangs wurden nur gesundheitsrelevante Sonderleistungen, besonders Lebensmittelzulagen, von ärztlichen Bescheinigungen abhängig gemacht, später immer häufiger auch Transportmöglichkeiten und der Erwerb von Gegenständen des täglichen Bedarfs wie Schuhwerk und Kleidung. ${ }^{17}$ Im November 1944 stellten deutsche Ärzte allein für zusätzliche Nahrungsmittel mehr als eine halbe Million Bescheinigungen aus. ${ }^{18}$

Darüber, wie Ärzte ihren kranken Patienten unter diesen Bedingungen gegenübertraten, wissen wir wenig. Auch wenn Hinweise dafür vorliegen, daß einige Ärzte, z.B. bei der Behandlung von Tuberkulosekranken und Alten, auf eigene Faust zwischen volkswirtschaftlich "nützlichen" und in ihren Augen weniger „nützlichen" Patienten unterschieden, ${ }^{19}$ scheint die Mehrzahl der Mediziner weiterhin „normal“ - d.h. im Sinne herkömmlicher ärztlicher Heilkunst - behandelt zu haben. Ein Großteil der Fälle, in denen sich Patienten über das Gesundheitswesen beklagten, kam dadurch zustande, daß zu wenig Ärzte verfügbar waren. Die Wartezeiten in überfüllten Sprechzimmern im Verein mit einer spürbar reduzierten Dauer des Arztkontaktes erzeugte bei vielen Patienten das Gefühl, schlechter als zuvor behandelt zu werden. Seit 1941 stieg die Zahl der überlastungsbedingten Kunstfehler deutlich, zudem führte das Fernbleiben von Ärzten auch bei akut behandlungsbedürftigen Krankheiten zu erheblicher Unruhe unter der Bevölkerung. ${ }^{20}$ Trotzdem prägte Normalität unter den eingeschränkten Bedingungen des Krieges die Mehrheit der Arzt-Patienten-Kontakte. Zwei Handlungsfelder, in denen die Funktionserwartungen des nationalsozialistischen Staates an Ärzte und Patienten mit deren traditionellen Verhaltensmustern in Konflikt gerieten, sind besonders geeignet, diese These zu überprüfen: die Einbindung von Medizinern in die Rassenpolitik des „Dritten Reiches“ und die Testatpraxis niedergelassener Ärzte.

Während sich ein erheblicher Teil der nicht katholisch gebundenen Ärzte bereitwillig an der Durchsetzung der nationalsozialistischen Erbgesundheitspolitik beteiligte, entzogen sich viele Mediziner den Versuchen des Regimes, den Zugang

15 Vgl. Tab. C.2; S. 192-200.

16 Boberach, SD-Berichte, 30. 4. 1942, Bd. 10, S. 3692 f.

17 Conti an Wächtler, 30.4 1942, StAB, M 30/846; Boberach, SD-Berichte, 6. 10. 1941, Bd. 8, S. 2832; 19. 1. 1942, Bd. 9, S. $3227 \mathrm{f}$.

18 Berechnet anhand der Kostenaufstellungen der Ärztekammern Baden, Westfalen-Nord und Westfalen-Süd für November 1944, BAB, R 18/3817 (ohne die Ärztekammern Sudetenland und Wartheland).

19 Vgl. S. 295, 300-304.

20 Exemplarisch: die Urteile des Ärztegerichtshofes gegen Dr. Helmut A. und Dr. Willi O., 10. 10. 1941, AKBV/134, die die bei Ohnmacht vorgeschriebenen Hausbesuche verweigert hatten; Boberach, SD-Berichte, 6. 10. 1941, Bd. 8, S. 2831; vgl. S. 196. 
zur Gesundheitsversorgung nach rassistischen Wertigkeitskriterien zu hierarchisieren. ${ }^{21}$ Zahlreiche Ärzte, in einigen Fällen auch prononcierte Nationalsozialisten, behandelten ihre jüdischen Patienten auch nach der nationalsozialistischen Machtergreifung, teilweise bis in die Kriegsjahre hinein.22 Umgekehrt hielten viele nichtjüdische Patienten an ihren jüdischen Ärzten fest, auch wenn sie deswegen, etwa als Beamte, erhebliche Nachteile zu befüchten hatten. ${ }^{23}$ Offenbar bildeten langfristig gewachsene Bindungen zwischen Ärzten und Patienten eine Barriere gegen das Eindringen nationalsozialistischer Maximen in das medizinische Alltagshandeln. Als während des Krieges feste Arztbezirke eingerichtet werden sollten, um das knappe Benzinkontingent der Landärzte rationeller zu nutzen, reagierte die Bevölkerung empört. Die „Einschränkung der freien Arztwahl und damit auch die Vernichtung des Gedankens des Hausarztes"24, so ein SD-Bericht vom Dezember 1942, habe „in der Bevölkerung starken Unwillen hervorgerufen“, und zu "einer unaufhörlichen Kette des Mißtrauens, der Unzufriedenheit und der Meckerei“ geführt, da „durch eine solche Maßnahme eine in vielen Fällen schon seit Jahrzehnten bestehende familiäre Betreuung durch den Hausarzt, wie sie gerade auf dem Lande und in den kleineren Städten auch heute noch üblich sei, unmöglich gemacht werde“. Auch dieser Protest kann als Indiz für das Weiterbestehen tradierter Arzt-Patienten-Bindungen gewertet werden.

$\mathrm{Daß}$ sich ärztliches Handeln damit allein nicht hinreichend erklären läßt, zeigt allerdings das Beispiel der Zwangsarbeiter, zu denen solche Solidarbeziehungen nicht bestanden. Auch wenn die Richtlinien des Regimes die medizinische Diskriminierung der Zwangsarbeiter vorschrieben und Patienten sich über deren Anwesenheit in den Sprechzimmern und Krankensälen beschwerten, 25 scheint die große Mehrheit der Ärzte in Einrichtungen des zivilen Gesundheitswesens solche Vorschriften nicht befolgt und diese Patientengruppe nach den Regeln der ärztlichen Kunst behandelt zu haben. ${ }^{26}$ Charakteristisch dafür, wie Amtsinhaber des nationalsozialistischen Gesundheitswesens auf solche Nachrichten reagierten, sind die Äußerungen des thüringischen KVD-Funktionärs und Gaugesundheits-

21 Etwa ein Siebtel der Sterilisationsanzeigen nach dem Gesetz zur Verhütung erbkranken Nachwuchses erfolgte durch Ärzte, die nicht im öffentlichen Gesundheitsdienst beschäftigt waren; Vossen, Gesundheitsämter, S. 244 f., dagegen finden sich bei Kersting, Anstaltsärzte, S. $265 \mathrm{f}$., Hinweise auf Formen passiver Verweigerung westfälischer Ärzte gegen die Mitwirkung an Zwangssterilisationen.

22 Zimmermann, Jena, S. 99f.; Forßmann, Selbstversuch, S. 211 f.; Urteil des Ärztegerichtshofes gegen Dr. Gustav F., 15. 11. 1940, AKBV/135; Spruchkammerverfahren Prof. Hermann W., BayHStA, MSo/1240.

23 Behnken, Sopade-Berichte 1935, Bd. 2, S. 115; Drecoll/Schleusener/Winstel, Verfolgung, S. $51 \mathrm{f}$.

24 Boberach, SD-Berichte, 17. 12. 1942, Bd. 12, S. 4579.

25 Stimmungsbericht der NS-Frauenschaft, Gau Essen, Anhang zum Tätigkeitsbericht der Reichsfrauenführung, Februar/März 1941, S. 46, BAB, NS 22/925. Unmittelbar davor war die gemeinsame Behandlung osteuropäischer Zwangsarbeiter mit deutschen Patienten aufgrund von Beschwerden aus der Bevölkerung untersagt worden; $\mathrm{BAB}$, Conti, Rundschreiben an die Ober- und Regierungspräsidenten, 27. 1. 1941, BAB, R 18/5576.

26 Boberach, SD-Berichte, 5. 3. 1942, Bd. 9, S. 3415; 30. 7. 1942, Bd. 11, S. 4026 f.; Bericht des SD-Leitabschnitts Breslau, 17. 10. 1944, ZAhdS, 500/3/764; Heusler, Arbeitseinsatz, S. 367. 
führers Rhode. Dieser beklagte, „daß verschiedene Berufskameraden bei der Behandlung von Kriegsgefangenen eine völlig unverständliche Haltung einnehmen ". ${ }^{27}$ Eine medizinische Vollversorgung dieser Patienten durch deutsche Ärzte sei nicht angängig, Krankenhausbehandlung käme nur bei Lebensgefahr in Frage. „Es müßte für jeden deutschen Arzt eine Selbstverständlichkeit sein, daß die ärztliche Behandlung bei Kriegsgefangenen auf das Maß des unbedingt Notwendigen zu beschränken ist." Noch stärker trat der Konflikt zwischen traditionellen berufsethischen Vorstellungen und den rassenpolitischen Postulaten des „Dritten Reiches" bei der Durchführung von Schwangerschaftsabbrüchen an russischen und polnischen Zwangsarbeiterinnen zu Tage. Da aufgrund des Arbeitskräftebedarfs schwangere Ostarbeiterinnen nicht mehr wie bisher in ihre Heimatgebiete zurücktransportiert werden sollten, hatte das NS-Regime im Frühjahr 1943 das bis dahin strikte Abtreibungsverbot gelockert und die Durchführung der Abbrüche in die Hände der regionalen Ärztekammern gelegt. ${ }^{28}$ Schwangerschaftsabbrüche galten jedoch für die Mehrzahl der Ärzte als berufsethisch nicht vertretbar. ${ }^{29}$ Daher hatte das Regime nicht nur in katholischen Regionen Schwierigkeiten, Ärzte zu finden, die den Eingriff durchführten. ${ }^{30}$

Ein zweites, aus der Sicht des Regimes womöglich noch wichtigeres Konfliktfeld bildete die medizinische Definitionsgewalt über die Scheidelinie zwischen krank und gesund und die daraus resultierenden Folgen. Die Politik der Reichsärzteführung war in diesem Punkt von erheblichem Mißtrauen gegenüber ihren in der Allgemeinpraxis tätigen Standeskollegen geprägt, sofern sich aus dem ärztlichen Urteil Folgen für die Zuteilung knapper Lebensmittel oder die Arbeitsfähigkeit der Patienten ergaben. Testate über medizinisch begründete Lebensmittelzulagen bedurften seit Kriegsbeginn der Gegenprüfung durch die Reichsärztekammer, die diese Aufgabe an ausgewählte Ärzte, zumeist örtliche Mitarbeiter des Hauptamtes für Volksgesundheit und Funktionäre der ärztlichen Bezirksvereinigungen, übertrug. ${ }^{31}$ Die wiederholten Reglementierungsanstrengungen und Verurteilungen von Ärzten, die gegen diese Bestimmungen verstoßen und teilweise ohne eingehende Untersuchungen zusätzliche Lebensmittel verordnet hatten, weisen jedoch darauf hin, daß Ärzte die vom NS-Regime vorgegebenen Beurteilungskriterien keineswegs durchgängig befolgten und auch Gefälligkeitsatteste

27 Rundschreiben der KVD-Landesstelle Thüringen 6/41 vom 25. 9. 1941, ThüHStA, KVD/ 6.

28 Conti an die Gauämter für Volksgesundheit, 11. 3. 1943, StAA, NSDÄB Augsburg-Land/ 1; Rundschreiben des RMdI an die leitenden Medizinalbeamten, 8. 7. 1943, NRWHStA, Regierung Düsseldorf/55185-II, Bl. 441.

29 Aus der Sicht der zeitgenössischen Medizinethik Ramm, Rechts- und Standeskunde, S. 101; Gruber, Ethik, S. 28 f.

30 Monatsbericht des Regierungspräsidenten von Oberbayern, 7. 6. 1944, S. 17, BayHStA, MA/106695; Flugplatzkommando Finsterwalde an das Gesundheitsamt Luckau, 22. 10. 1943, BrLHA, Br. Pr. Rep. 45 D/185.

31 Von 20 Prüfungsbeauftragten für Atteste über Zusatzlebensmittel im Gebiet der Ärztekammer Thüringen waren 11 Mitarbeiter der NSDAP-Ämter für Volksgesundheit, vier amtierten als Vorsitzende der ärztlichen Bezirksvereinigungen; Verzeichnis der Genehmigungsstellen für die Gewährung von Lebensmittel-Zulagen wegen Krankheit im Bereich der Ärztekammer Thüringen, September 1939, ThüHStA, KVD/170-72. 
nicht eben selten waren. ${ }^{32}$ Deutlicher noch gilt dies für den zentralen Bereich der gesundheitsbedingten Arbeitsunfähigkeit. Vertreter der Rüstungswirtschaft, der Arbeitseinsatzbehörden und des SD sahen in der „reichlich weiche(n) Beurteilung seitens der meisten Ärzte" 33 die Hauptursache der seit Kriegsbeginn steigenden Krankenstände. Besonders Ärzte in der freien Praxis, so die Beschwerde, zögen sich hinter ihre Verantwortung für die Gesundheit der Patienten zurück und zeigten „Hemmungen [...] von sich aus, so wie es bei der außerordentlich angespannten Arbeitsmarktlage notwendig wäre, durchzugreifen". ${ }^{34}$ "Jeder der sich um die Arbeit drücken will", so klagte die Leitung eines chemischen Großbetriebs in Brandenburg, „läuft zum Arzt, weil er genau weiss, dass ihm der Arzt alles bescheinigt, was er für seine Drückerei braucht" ${ }^{35}$ Insbesondere Frauen könnten mit Bestimmtheit damit rechnen, krank geschrieben zu werden oder einige Tage Schonung verordnet zu bekommen. ${ }^{36}$ Zur Behebung dieses aus ihrer Sicht unhaltbaren Zustands begnügte sich die Reichsärzteführung nicht mit kollegialen Appellen. In zunehmend kürzeren Abständen, seit 1943 bereits drei Tage nach der Krankschreibung, überprüften Vertrauensärzte das Urteil des behandelnden Arztes. ${ }^{37}$ Gauamtsleiter des Hauptamtes für Volksgesundheit bedrohten Mediziner, die bei der Ausstellung von Attesten nicht die "notwendige Härte" 38 an den Tag legen wollten, mit Approbationsentzug, Geld- und Freiheitsstrafen. Trotzdem, so klagte ein Vertreter der Landesversicherungsanstalt Oberbayern, zeigten alle Versuche, die Einstellung der Ärzte zu ändern, „leider nur vorübergehende Erfolge". 39

Insgesamt deuten diese Befunde eher auf eine niedrige Eindringtiefe nationalsozialistischen Gedankengutes in die ärztliche Praxis hin. Dies läßt sich nur schwer mit der bekanntermaßen hohen Affinität von Medizinern zur Hitlerpartei und der großen Zahl der NS-Verbrechen, an denen Ärzte beteiligt waren, in Einklang

32 Urteil des Ärztegerichtshofes gegen Dr. Hugo R., 21. 10. 1942, AKBV/134; Urteil des Ärztegerichtshofes gegen Dr. Josef B., 3. 6. 1943, ebenda. In beiden Fällen wurden Ärzte wegen der leichtfertigen Ausstellung von Zeugnissen für Lebensmittelzulagen für Schwangere verwarnt.

33 Bericht des Referenten des Beauftragten des Reichsministeriums für Bewaffnung und Munition im Wehrkreis XII, o.D., Abschrift als Anlage zum Schreiben der KVD Baden-Elsaß an das Hauptamt für Volksgesundheit, 11. 5. 1942, BAB, R 18/3758.

34 Betriebskrankenkasse der Mitteldeutschen Stahlwerke AG an die Landesstelle Sachsen des Reichsverbandes der Betriebskrankenkassen, 30.5. 1944, SächsHStA, Stahl- und Walzwerk Riesa/20.19 (Zitat); SD-Leitabschnitt München, Bericht über Produktionsausfall durch Krankheit und Urlaub, 31. 7. 1944, ZAhdS, 500/3/764.

35 Aktenvermerk der kaufmännischen Abteilung der Kurmärkischen Zellwolle u. Zellulose AG/Wittenberge, 25. 5. 1940, BrLHA, Br. Pr. Rep. 75/53.

36 Bericht des SD-Leitabschnitts Breslau, 17. 10. 1944, ZAhdS, 500/4/74; SD-Leitabschnitt München, Bericht über Produktionsausfall durch Krankheit und Urlaub, 31.7. 1944, ebenda.

37 Vgl. S. 246.

38 Klinger, Wege, S. 100 (Zitat); Rundschreiben des Gauamtes für Volksgesundheit Bayerische Ostmark, I. Vierteljahr 1943, abgedruckt im Bericht des SD-Abschnitts Bayreuth vom 27. 4. 1943, StAB, M 30/1043.

39 Landesversicherungsanstalt-Oberbayern an das Reichsversicherungsamt, Abteilung Kranken- und Invalidenversicherung, 14. 11. 1942, BAB, R 89/5372, Bl. 135. 
bringen. Eine mögliche Erklärung für diese Diskrepanz besteht darin, daß vielen Ärzten die Nagelprobe der Aufgabe des Nicht-Schaden-Prinzips schlichtweg erspart blieb. Der von Ernst Fraenkel beschriebene „Doppelstaat“ in seiner Verschränkung von nationalsozialistisch durchdrungenen, kriminalisierten Lebensbereichen und Zonen der Normalität, die zur Stabilisierung der Herrschaftsordnung unerläßlich waren, war auch im Gesundheitswesen anzutreffen. Spezifisch nationalsozialistische Medikalisierungsstrategien wie die Zwangssterilisationen oblagen zumeist dem öffentlichen Gesundheitsdienst und waren als Maßnahmemedizin dem Handlungsfeld des praktischen Arztes weitgehend entzogen.

Eine weitere mögliche Erklärung für die Stabilität des Arzt-Patienten-Verhältnisses über den politischen Umbruch hinweg gründet in der fortgesetzten Bedeutung merkantiler Erwägungen für das ärztliche Handeln. Solange ein halbwegs freier Markt für medizinische Dienstleistungen bestand, waren niedergelassene Ärzte auf die Zufriedenheit der Behandelten, auf die Wiederkehr ihrer Patienten angewiesen. Gerade die spezifisch nationalsozialistischen Ausprägungen der $\mathrm{Me}-$ dizin, etwa die "Neue Deutsche Heilkunde“, wurden von der Bevölkerung nicht akzeptiert und Ärzte, die dezidiert im Sinne des Regimes praktizierten, wenn irgend möglich, von den Kranken gemieden. ${ }^{40}$

Der nationalsozialistische Umgestaltungsanspruch wurde zudem entscheidend begrenzt durch das aus der Sicht des Regimes ungünstige Verhältnis zwischen seiner Fähigkeit, kurzfristige Veränderungen herbeizuführen, und dem stabilen beruflichen Selbstbild der praktizierenden Ärzte, die ausgesprochen sensibel auf Versuche medizinfremder Instanzen reagierten, Zugriff auf die individualisierte Beziehung zwischen Arzt und Patient zu erlangen. Der alltägliche Umgang des Arztes mit seinem kranken Patienten war das Produkt einer professionellen Sozialisation von hoher Prägekraft, die sich schnell wirksamen Umgestaltungsversuchen weitgehend entzog. Das hohe Durchschnittsalter der im Zivilleben verbliebenen Mediziner wirkte den vom NS-Regime beabsichtigten Veränderungen entgegen, so daß der Krieg hier nicht als Katalysator von Veränderungen, sondern eher als Ursache der Beharrung wirkte. Etwa die Hälfte der während des Krieges in Deutschland praktizierenden Ärzte hatte das 50. Lebensjahr bereits überschritten und war noch im Kaiserreich und den Anfangsjahren der Weimarer Republik professionell sozialisiert worden. ${ }^{41}$ Gerade diese „älteren Herren der Ärzteschaft" 42 galten als besonders entgegenkommend gegenüber ihren Patienten. Ihr berufliches Selbstverständnis basierte zwar auf dem Modell einer stark hierarchisch geprägten Arzt-Patienten-Beziehung, diese blieb allerdings weiterhin an das Ideal ärztlicher Hilfe für den einzelnen Kranken gebunden und dem Zugriff des Staates weitgehend entzogen. Die Krankenhilfe orientierte sich an einem über Jahrzehnte hinweg eingeschliffenen medizinischen Erfahrungswissen, das festge-

40 Kudlien, Fürsorge, S. 103; Rüther, Standeswesen, S. 187.

41 Tab. C.2.

42 Landesversicherungsanstalt Sachsen an die Landesgeschäftsstelle Sachsen des Reichsverbandes der Betriebskrankenkassen, 5. 6. 1944, Anlage C, Anregungen zum Krankenstand, SächsHStA, Stahl- und Walzwerk Riesa/20.19 (Zitat); Bericht des SD-Leitabschnitts Breslau, 17. 10. 1944, ZAhdS, 500/4/74. 
fügte Vorstellungen über angemessene Therapien, erforderliche Schonungszeiten für Kranke und medizinisch begründete Diätvorschriften enthielt, und sich gegenüber nationalsozialistischen Abänderungsversuchen als weitgehend resistent erwies. Die traditionell hohe, in den Kassenkämpfen der Weimarer Republik politisierte Bedeutung, die Ärzte ihrer professionellen Autonomie beimaßen, stabilisierte den etablierten Kanon medizinischer Therapie. ${ }^{43}$ Bei jüngeren Ärzten, die ihre berufliche Sozialisation in den späten zwanziger und frühen dreißiger Jahren durchliefen, als sich die ökonomische Depravation der Ärzteschaft mit einer Krise des beruflichen Selbstverständnisses paarte, scheint die Bereitschaft, sich auf den vom Nationalsozialismus propagierten Wandel einzulassen, und diese Art der Behandlung auch auf den Normalpatienten zu übertragen, im Durchschnitt größer gewesen zu sein. Entsprechend häufiger waren die Klagen der Patienten über Angehörige dieser Alterskohorte.

Insgesamt gesehen also kaum nennenswerte Veränderungen? Wohl eher nicht. Längst nicht alle Ärzte entzogen sich den nationalsozialistischen Umgestaltungsplänen. Die universitäre Forschung zeigte sich zum Beispiel sehr empfänglich für die neuen politischen Vorgaben, indem sie immer mehr Krankheiten erbbiologisch interpretierte. ${ }^{44}$ Inwieweit solche Konzepte aus der medizinischen Diskussion in die Praxis der Normalmedizin vordrangen, läßt sich nur schwer bestimmen. Sicher ist, daß ihr Eindringen in die vom staatlichen Gesundheitswesen betriebene „Medizin gegen Unbrauchbare“ 45 zur hunderttausendfachen Verletzung des für die Arzt-Patienten-Beziehung konstitutiven Nicht-Schaden-Prinzips geführt hat. Die mehr als 360000 Zwangssterilisationen wurden in der Regel gegen den Willen der Patienten ausgeführt und hatten besonders bei Frauen nicht selten den Tod der Operierten zur Folge. Die Operateure waren in der Mehrzahl Ärzte der Approbationsjahrgänge nach 1930, für die der Eingriff zu den Routinetätigkeiten im Rahmen ihrer medizinischen Ausbildung gehörte. ${ }^{46}$

Daß sich das Verhältnis zum Arzt im Verlauf des Krieges für die Patienten spürbar wandelte, hängt vor allem mit zwei vom NS-Regime gezielt herbeigeführten Veränderungen zusammen, die das Gesicht der zweiten Kriegshälfte prägten: dem Ausbau der Kontrollmedizin und der Verlagerung der medizinischen Primärversorgung von den niedergelassenen Ärzten zu Betriebsärzten und Ärzten des öffentlichen Gesundheitsdienstes. In den Gesundheitsämtern, den vertrauensärztlichen Dienststellen und besonders bei den Betriebsärzten war der Anteil jüngerer, nationalsozialistisch engagierter und im Sinne des Regimes praktizierender Ärzte besonders groß. ${ }^{47}$ Hierdurch erhöhte sich für den Kranken das Risiko, von einem nicht mehr zum Wohle des Patienten praktizierenden Arzt behandelt zu werden,

43 Kater, Professionalisation, S. 680, 684; Thomsen, Ärzte, S. 59, 63; Wolf, Interessen, S. 109.

${ }^{44}$ Hahn, Entwicklungstrends, S. 116; zum Gesamtproblem vgl. Kater, Doctors, S. 111-150.

45 In Anlehnung an Aly, Medizin.

46 Pfäfflin u. a., Krankenversorgung, S. 286; Zimmermann, Jena, S. 146-155.

47 Für den vertrauensärztlichen Dienst vgl. Behnken, Sopade-Berichte 1936, Bd. 3, S. 637 f. Der NSDAP-Anteil unter den Betriebsärzten war mit 81,5 Prozent erheblich höher als der des Gesamtdurchschnitts aller Ärzte (44,8 Prozent); Namenskartei der Betriebsärzte im Gau Thüringen [1943], BAB, NS 37/1065; Kater, Doctors, S. 245. 
erheblich. Die meisten bekannten Fälle ärztlicher Insensibilität und eindeutig patientenschädigenden Verhaltens stammen aus diesen Bereichen.

Schließlich veränderte sich das gesundheitspolitische Bezugssystem, in das die Arzt-Patienten-Beziehungen eingebettet waren, während des Krieges entscheidend, denn auch das medizinische Alltagshandeln fand in einem zunehmend verbrecherischen gesundheitspolitischen Kontext statt, dessen Umrisse seit dem Sommer 1941 zusehends erkennbar wurden. Dies wird deutlich, wenn man den Blick auf die Bewertung des Gesundheitssystems durch die Patienten richtet. Bei einer Umfrage, die die Gesellschaft für Konsumforschung in den Jahren 1938/39 bei etwa 10000 Personen durchgeführt hatte, gaben rund 70 Prozent aller Befragten an, daß sie Vertrauen zu ihrem Hausarzt hätten. Rechnet man aus diesem Ergebnis die Anhänger alternativer Heilmethoden heraus, so ergibt sich für die Zeit vor dem Beginn des Zweiten Weltkrieges eine hohe Zustimmung der Bevölkerung zu ihren Ärzten. Die Anzahl der „Arztgegner aus Erfahrung“, also derjenigen Personen, die Negativerlebnisse mit dem Gesundheitswesen gemacht hatten, war demgegenüber mit etwa einem Prozent der Befragten vergleichsweise gering. ${ }^{48}$

Für die Kriegszeit fehlen vergleichbare Umfragen, doch deuten zahlreiche Indizien darauf, daß das Vertrauen der Bevölkerung zu ihren Ärzten rapide schwand. ${ }^{49}$ Dieser Vertrauensschwund war eingebettet in einen allgemeinen Ansehensverlust gesundheitspolitischer Einrichtungen, der in einem unmittelbaren Zusammenhang zu der Ausgrenzung von Teilen der Bevölkerung aus der medizinischen Versorgung und dem massenhaften Mord an den Insassen der Heil- und Pflegeanstalten stand. Waren die Zwangssterilisationen als Maßnahme gegen eine vergleichsweise klar umrissene Gruppe der Bevölkerung außerhalb des katholischen Milieus kaum auf Widerstand gestoßen, so verbreitete sich nach dem Bekanntwerden der „Euthanasie“-Morde zunehmend die Sorge, daß nicht nur Geisteskranke, sondern prinzipiell jeder Arbeitsunfähige von solchen Maßnahmen betroffen sein könne. Zunächst richtete sich das Mißtrauen der Bevölkerung gegen die Heil- und Pflegeanstalten, deren Klienten sich kaum mehr freiwillig in ihre Obhut begaben und dort, wo die Möglichkeit bestand, vermehrt auf konfessionelle Anstalten auswichen. ${ }^{50}$ In einem zweiten Schritt gerieten auch diejenigen Angebote der Gesundheitsfürsorge unter Verdacht, die als besonders parteinah galten. In Baden und in Westfalen weigerten sich Teile der Bevölkerung, an der vom Hauptamt für Volksgesundheit mit Personal der SS durchgeführten Röntgen-Reihen-Untersuchung zur Tuberkulosefrüherkennung teilzunehmen, da sie befürchteten, im Falle eines positiven Befundes ebenfalls ermordet zu werden. ${ }^{51}$

48 Schultz/Bergler/Michl, Vertrauen, S. $34 \mathrm{f}$.

49 Boberach, SD-Berichte, 6. 10. 1941, Bd. 8, S. 2831.

50 Reichsarbeitsgemeinschaft für Heil- und Pflegeanstalten, Zusammenfassender Bericht über das Ergebnis der Berichte der Oberpräsidenten, Landesregierungen usw. zum Rundschreiben des Reichsinnenministeriums vom 6. 1. 1942 IV g 8410/41 - 5114, 11.7. 1942, $\mathrm{BAB}, \mathrm{R}$ 96-I/2.

51 Stimmungsbericht der NS-Frauenschaft, Gau Baden, Februar/März 1941, S. 30, Anhang zum Tätigkeitsbericht der Reichsfrauenführung, BAB, NS 22/925; Kreisleitung Tecklenburg an den Stellvertretenden Gauleiter des Gaues Westfalen-Nord, 11. 9. 1941, StAMü, Kreis- und Ortsgruppenleitungen/125. 
Zuletzt schließlich sprang die Vertrauenskrise der gesundheitspolitischen Institutionen auch auf die niedergelassenen Ärzte über. Im Frühjahr 1944 liefen in der Berliner Zentrale des SD aus dem ganzen Reichsgebiet Meldungen zusammen, die auf eine zunehmende Zerrüttung des Vertrauensverhältnisses zwischen Arzt und Patient durch die medizinischen Staatsverbrechen des „Dritten Reiches“ schließen lassen. Vor allem ältere Patienten vermieden Arztbesuche oder verweigerten die Einnahme verordneter Arzneien, da sie fürchteten, Ärzte würden im Regierungsauftrag auf ihren vorzeitigen Tod hinwirken.52

52 Ohlendorf, Rundschreiben an die SD-(Leit)Abschnitte und Hauptaußenstellen, 20. 3. 1944, ZAhdS, 500/4/330. 